\title{
Clinica Dermosifilopatica della R. Universita di Roma.
}

\author{
Mai 1914.
}

Campana, Roberto. Weiteres über die Sporozoen des Molluscum contagiosum (Bemerkungen aus der Sitzung der Dermatologischen Gesellschaft vom 19. Dezember 1913). p. 33.

Bemerkungen $\mathrm{zu}$ einer Demonstration mikroskopischer Präparate von della Favera, und Hinweis auf eine Arbeit aus dem Zentralblatt für Bakteriologie, Band LXXIII, p. 137.

Paolo, Alessi. Die Eigenheiten des Verhaltens des Salvarsans bei Syphilitikern und bei tierischen Geweben. p. 27.

Die ersten Abschnitte der Arbeit beschäftigen sich mit klinischen Beobachtungen. Es folgen experimentelle Untersuchungen und die histologischen Befunde am Nervensystem mit Salvarsan behandelter Meerschweinchen.

Campana, R. Studien über Vakzinationengegen Typhus. p. 61 .

Costa, Tito. Veränderungen der Haut und anderer Organe in ihrem morphologischen Verlaufe studiert und beschrieben. p. 63.

Nicht zum kurzen Referat geeignet.

Campana, R. Tuberkulose (Fieber etc.). p. 79.

Fritz Juliusberg (Posen).

\section{The British Journal of Dermatology.}

\section{April 1914.}

Sibley, W. Knowsley. Ein Fall von rezidivierender bullöser Eruption. p. 115.

Die 63jährige Patientin leidet seit 30 Jahren an einer fortwährend rezidivierenden Eruption an der Vorderseite beider Beine, seit 10 Jahren an einem ähnlichen Zustand an der Hinterseite des linken Arms. Die Affektion schloß sich am Bein an einen Schlag an. Seitdem entstehen fortwährend Blasen, welche zu starken Blutungen Anlaß geben. Vor zwei Jahren wurde eine harte hornige Wucherung von der Vorderseite des rechten Beines entfernt. Die Haut an den Beinen weist neben einigen frischen Blasen Pigmentationen und oberflächliche Narben auf. Der Zustand der Patientin erinnert an die Epidermolysis bullosa, doch bestand die Affektion nicht in den frühen Lebensjahren; es findet sich kein Anhaltspunkt für eine Heredität und die Finger und Nägel sind frei.

Fritz Juliusberg (Posen). 